\title{
Epidemiologische Untersuchungen zur Frage der Phthisiogenese.
}

Von

Kreisarzt Dr. Hillenberg,

Zeitz.

Welche Bedeutung der Kindheitsinfektion für die Entstehung der Lungenschwindsucht Erwachsener von vielen Arzten heutzutage beigelegt wird, auf welchen Grundlagen diese moderne Lehre der Phthisiogenese aufgebaut worden, welche Forscher sie begründet und entwickelt haben, ist ja bekannt, so daB ich bei meinen kurzen Darlegungen hierauf nicht einzugehen brauche. Trotz des scheinbar völligen Ineinandergreifens aller Argumente und der scheinbaren Lückenlosigkeit des Beweismaterials für diese Lehre, trotz des Bestechenden der grundlegenden Experimente und ihrer Ergebnisse weisen letztere nach meinem Dafürhalten die eine Lücke auf, daß sie sich mit epidemiologischen Erfahrungen und Tatsachen nicht in Einklang bringen lassen. Hierauf ist von mir bereits auf der zehnten internationalen Konferenz in Rom hingewiesen worden. Später haben die beiden Breslauer Arrte Bruck und Steinberg ${ }^{1}$ eine bedeutsame Arbeit über „Die Verbreitung der Lungentuberkulose in Breslauer Familien, Wobnungen und Werkstätten" veröffentlicht, in der sie zu dem SchluB kommen, daß „der jetzige Stand der Forschung die Möglichkeit sowohl ,endogener" wie ,exogener" Schwindsuchtsentstehung zuläBt". Auch vom pathologisch-anatomischen Standpunkt vermag neuestens ein solch hervorragender Gelehrter wie J. Orth der modernen Auffassung der Schwindsuchtsentstehung nicht beizupflichten, wie er in einem in der Gesamtsitzung der Königlich PreuBischen Akademie der Wissenschaften vom 16. Januar d. J. gehaltenen Vortrag dargetan. Er gelangt hier zu der

${ }^{1}$ Diese Zeitschrift. Bd. LXXI. 1912. 
Schlubfolgerung: ,Die pathologisch-anatomische Erfahrung widerspricht 1. der Annahme, daB jede Lungensehwindsucht Produkt einer Reinfektion sein müsse und 2. der Behauptung, daB nur eine massire Autoreinfektion geeignet wäre, Lungenschwindsucht zu erzeugen".

Es ist freilich nicht zu bezweifeln, daB Jugendinfektionen die Basis für Lungenerkrankungen des späteren Lebensalters abgeben können, und ich meine, man hat seit der Entdeckung des Tuberkelbacillus für gewisse Fälle von Phthisen jüngerer Erwachsener, d. h. solche, deren Träger aus tuberkulösen Familien stammten, stets die Ansteckung in die Jugendzeit der betreffenden Individuen verlegt und die in späteren Jahren erfolgende Erkrankung als den wahrscheinlichen AbschluB einer vor Jahren in der Familie erfolgten Infektion gehalten. Auch darin hat Römer wohl recht, $\mathrm{da} b$ eine Nachinfeltion in einem bereits infizierten menschlichen Organismus anders als in einem von Tuberkulosevirus noch verschonten Körper verläuft, sowie darin, daB das Überstehen einer leichten Infektion eine erhöhte Widerstandsfāhigkeit gegen Reinfektion verleiht. Die sehr groBe Zahl der Infizierten, bei der freilich der Anteil des Perlsuchtbacillus von den Autoren meist unberücksichtigt gelassen wird, die ausgedehnte Gelegenheit zu Reinfektionen und die im Vergleich hierzu kleine Ziffer der später wirklich Erkrankten spricht - vom Tierexperiment und sonstigen biologischen Erfahrungen ganz abgesehen - für das Statthaben einer Immunisierung, gegen die allerdings Orth wieder sehr triftige Gründe ins Feld führt, auf die hier nicht näher eingegangen sei. Nur die praktisch so bedeutsame weitere Folgerung, daB die Lungenschwindsucht in der Regel auf „metastasierende Autoinfektion“ zurückzuführen sei, ist meines Erachtens bisher noch durch kein Tierexperiment, keine sonstige Tatsache einwandfrei erhärtet, kann auch, wie ich schon früher betont, auf tierexperimentellem Wege gar nicht bewiesen werden, da die Autoreinfektion und ihre Bedeutung beim Tier wohl auBerhalb jeglicher Beobachtungsmöglichkeit liegt, und die vielgenannten kavernösen Lungenveränderungen bei reinfizierten Tieren eben durch exogene Infektionen hervorgerufen worden sind.

Am aussichtsreichsten, das Problem der Phthisiogenese seiner Lösung näher zu führen, sind meines Erachtens eingehende epidemiologische Untersuchungen, wie sie Bruck und Steinberg angestellt haben, denen in der Klientel der Breslauer Lungenfürsorgestelle ein vorzüglich zu bearbeitendes Material zu Gebote stand.

Für mich, der ich durch die Veröffentlichung der genannten Autoren zu gleichen Untersuchungen angeregt wurde, war die Erlangung einigermaßen zuverlässiger Unterlagen erheblich schwieriger, zumal es mir darauf 
ankam, ein noch umfangreicheres und dabei doch gut geklārtes Material zur Verfügung zu bekommen. Ich ging nun folgendermaßen vor: Es wurden an sämtliche Schulkinder aller im Stadt- und Landkreis Zeitz rorhandenen Schulen, ferner an die der beiden Volksschulen und der Bürgerschule des Nachbarkreises WeiBenfels Stadt, sowie schlieBlich in meinem Landkreise durch Schulkinder auch an solche Familien, die keine schulpflichtigen Kinder hatten, ausführliche Fragebogen zweeks Beantwortung durch die Angehörigen verteilt, die ausgefüllt wieder zur Schule mitgebracht, von den Lehrern durchgesehen und hier und da durch wertrolle Mitteilungen ergänzt wurden. Ihre Gesamtzahl betrug 14000 . Diese Fragebogen sollten mir ausschlieblich als Unterlagen für weitere Ermittelungen dienen. Ferner bat ich sämtliche Geistliche, mir Auszüge aus den Kirchenbüchern über alle seit dem Jahre 1880 angeblich an Schwindsucht Verstorbenen ihrer Sprengel mit Angabe von Alter, Todesjahr und verwandtschaftlichen Beziehungen zukommen zu lassen; auch dieser Bitte wurde ohne Ausnahme in liebenswürdiger Weise unter häufiger Hinzuf ügung wichtiger Notizen entsprochen. Diese Übersichten hatten lediglich den Zweck, mir über Alter und Todesjahr der einzelnen Verstorbenen, worüber die Angehörigen natürlich nicht immer zutreffend orientiert waren, zuverlässige Auskunft zu geben. Im Stadtkreis Zeitz bildeten die ärztlichen Todesbescheinigungen seit dem Jahre 1906 mir weitere Kontrollunterlagen.

Die Fragebogen enthielten folgende Fragen:

1. Sind in Ihrer engeren und weiteren Familie Fälle ron Lungentuberkulose (Schwindsucht, Auszehrung) vorgekommen?

a) bei den GroBeltern? b) Eltern? c) beim anderen Ehegatten (Mann, Frau)? d) bei den Kindern? e) bei sonstigen Verwandten? Möglichst genaue Angaben erbeten!

2. Hat die verstorbene Person als Kind oder in späteren Lebensjahren Gelegenheit gehabt, in der Nähe eines Schwindsüchtigen zu leben oder zu arbeiten?

3. Oder bestand gar keine nachweisbare Gelegenheit zum Verkehr mit einem Schwindsüchtigen, d. h. zur Ansteckung?

4. Welches Lebensalter hat der Verstorbene erreicht? In welchem Jahr, welchem Ort, welcher Wohnung (Straße, Hausnummer, Stockwerk) ist er verstorben?

5. Sind in dem betreffenden Hause, der Wohnung, der Nachbarschaft schon früher Fälle von Schwindsucht vorgekommen? Welche? Wann? Wie viele? 
6. Welchen Beruf hatte die verstorbene Person bzw. deren Vater? Genaue Angabe der Beschäftigung erbeten. Bei Erwerbstätigen: Wo hat sie gearbeitet?

7. Wie viele Geschwister hat die verstorbene Person gehabt? Wie viele von diesen haben gleichfalls in der Umgebung von Schwindsüchtigen gelebt, ohne später erkrankt zu sein? Wie alt sind sie geworden bzw. sind sie jetzt?

8. Beim Tode beider Ehegatten: Wie viel Zeit lag zwischen dem Tode des einen und der Erkrankung des andern?

9. Beim Tode eines Ehegatten: Ist der andere gesund geblieben?

10. Beim Tode oder bei Erkrankung von Kindern an Schwindsucht und vorhergegangener Schwindsucht der Eltern: Wie viel Zeit lag zwischen dem Tode a) des Vaters, b) der Mutter und dem Tode des Kindes bzw. der Kinder?

Name und Stand:

Wohnort:

StraBe und Nr.:

Ich erhielt auf diese Weise Kenntnis von rund 1000 Tuberkulosefällen, in der überwiegenden Mehrzahl Todesfälle, in der Minderzahl Erkrankungen noch Lebender betreffend, die jedoch nicht in dieser Zahl verwertet werden konnten; einmal war eine Reihe von Fragebogen allzu mangelhaft ausgef üllt, so daß sie nicht einmal Anhaltspunkte für weitere Nachforschungen darboten, sodann mußte eine Anzahl solcher Fälle aus. scheiden, in denen das Anstellen der nötigen Ermittelungen mit erheblichen Schwierigkeiten verknüpft war, bzw. die Angehörigen eingehendere Auskunft rerweigerten. So blieben für die weitere Nachforschung 702 Todes- bzw. Erkrankungsfälle übrig, bezüglich deren zum bei weitem größeren Teil von mir persönlich, zum kleineren mit Hilfe von Gemeindeschwestern, Lehrern und Geistlichen, denen ich die Fragebogen mit exakter Fragestellung übermittelte, möglichst genaue Aufklärung über die einzelnen Fälle, namentlich hinsichtlich der Infektionsquellen, zu erlangen versucht wurde. Daß schlieBlich die Mithilfe von Ärzten und Krankenhäusern zur Klarstellung unsicherer Fälle erbeten und meist in liebenswürdiger Weise gewährt wurde, sei noch ebenso betont wie der Umstand, daß irgendwie zweifelhafte Fälle in der Bearbeitung unberücksichtigt blieben. Auf diese Weise glaube ich unter Aufwendung erheblicher Mühe ein verhältnismäßig zuverlässiges Material erhalten zu haben, das zur epidemiologischen Prüfung der Frage der Phthisiogenese wohl brauchbar sein dürfte. Es ist ohne weiteres zuzugeben, daß dasselbe seine Mängel 
hat, die vornehmlich darin beruhen, daß der Untersucher in den allermeisten Fällen auf anamnestische Angaben von Laien angewiesen war, die ja stets mit einer gewissen Vorsicht zu bewerten sind. Mag darum auch dieser und jener Fall in Wirklichkeit keine Tuberkulose gewesen, mögen vor allem die Angaben über die Infektionsquelle nicht überall der Wirklichkeit entsprechen, so glaube ich doch, daB diese Fehler das Endergebnis nicht nennenswert beeinflussen dürften. Bevor ich dieses kurz mitteile, möchte ich noch bemerken, daB die Sterbeziffer an Tuberkulose wie im ganzen Regierungsbezirk Merseburg so auch in meinen Kreisen eine sehr niedrige ist, im Landkreise wohl die niedrigste der ganzen Monarchie: 9 auf 10000 Lebende, im Stadtkreise 11 auf 10000. Hierdurch hatte ich den Vorteil, den Zusammenhängen zwischen einzelnen Fällen mit großer Sicherheit nachgehen und ihre Quellen feststellen zu bönnen.

Trotzdem ist die Zahl derjenigen Fälle, in denen eine sichere Infektionsquelle nicht nachweisbar war, mit $412=58.6$ v. $\mathrm{H}$. eine recht groBe; was nicht zu verwundern ist, da es sich z. T. um Todesfälle handelt, die bereits 2 bis 3 Jahrzehnte zurückliegen; nähere Aufklärung war über sie trotz aller Bemühungen nicht immer zu erlangen. Vou diesen Fällen abgesehen, sind es Einzelerkrankungen jüngeren Datums, rein sporadische Fälle in absolut gesunden Familien, in denen Eltern und häufig eine große Zahl von Geschwistern gesund geblieben waren. Aber auch hinsichtlich dieser Gruppe haben wir noch zu unterscheiden zwischen solchen, in denen im Beruf, in der Beschäftigung des Verstorbenen wenigstens ein schädigendes Moment auffindbar war, und denjenigen, für die überhaupt jeder Anhaltspunkt für die Infektionsquelle fehlt bzw. als ausgeschlossen anzusehen ist, daß ein längeres bewußtes Zusammenarbeiten mit einem Schwindsüchtigen vorgelegen hat. Gerade diese Kategorie von Erkrankungen werden die Anhänger der Lehre von der infantilen Entstehung der Phthise auf Autoreinfektion zurückführen. Es könnte ihnen beigestimmt werden, zumal, soweit die Erkrankungen in engerem oder weiterem Anschlub an die Pubertät zum Ausbruch gekommen sind; aber man sieht sich dann meines Erachtens sofort vor die Frage gestellt: Warum kommt es nach leichten Frühinfektionen, die doch eigentlich immunisieren sollen - sogenannten schweren Infektionen waren jà die Betreffenden in ihrer Jugend nicht ausgesetzt gewesen -, relativ häufig zu tödlichen Phthisen, ohne daB besondere Schädigungen der betreffenden Individuen nachweisbar gewesen sind. Auf der anderen Seite können die Verfechter der alten Lehre von der exogenen Entstehung sagen: Da endogene'Entwicklung sich in der Regel nach schweren sogenannten familiären Infektionen finden soll, letztere hier jedoch nicht 
in Betracht kommen, so bleiben nur zwei Möglichkeiten übrig: entweder genügt auch jede leichte. Frühinfektion für spätere maligne Selbstinfektionen, wofür jene Fälle sprechen können, in denen ohne nachgewiesene schwere Familieninfektion sich meist im AnschluB an eine tiefer greifende Schädigung des jugendlichen Organismus eine Kette von verdächtigen Krankheitssymptomen anschliebt, deren letztes 'Glied eine manifeste Lungenschwindsucht ist; oder der phthisische Ausgang des Prozesses ist auf Infektion $v 0$ in a $u B$ en zurūckzuführen. Ich muB gestehen, daB mir die Entstehung dieser Fälle nicht völlig geklärt ist.

Auf die verschiedenen Altersklassen verteilen sich die genannten Einzelerkrankungen folgendermaßen:

$1-5 \quad 6-10|11-15| 16-20|21-25| 26-3031-3536-40|41-45| 46-55556-6566-70$ Jahre Jahre Jahre Jahre Jahre Jahre Jahre Jahre Jahre Jahre Jahre Jahre

\begin{tabular}{l|l|lllll|l|l|l|l|l}
3 & 2 & 4 & 19 & 18 & 31 & 30 & 36 & 27 & 27 & 16 & 5 \\
\hline 9
\end{tabular}

Ein gewisses Interesse erheischen die im Kindesalter zur Beobachtung gelangten Todesfälle. Worauf sind sie zurückzuführen? Hustende Phthisiker haben nach meinen Ermittelungen in der Nähe der fraglichen Kinder nicht gelebt; daB diese am dritten Orte Tuberkelbazillen aufgenommen hätten, ist wenigstens für die ganz jungen Kinder nicht recht wahrscheinlich, da sie aus ihrer gewohnten Umgebung wenig oder gar nicht herausgekommen sind. Da auch eine eigentliche ubiquitäre Verbreitung der Tuberkelbazillen abzulehnen ist, bleibt nur eine Infektion mit Perlsuchtbazillen übrig, für die ein Beweis natürlich nicht zu erbringen ist.

Außer diesen 218 gewissermaßen in der Luft schwebenden Einzeltodesfällen sind ihrer eine Reibe ermittelt worden, bei denen die betreffenden Individuen durch ihre soziale Stellung, durch den Ort ihrer Tätigkeit zum Teil zur Phthise disponiert, zum Teil der Gefahr der Infektion ständig ausgesetzt waren. Es handelt sich da zunächst um 13 Todesfälle von Stein- und Holzbildhauern und Zigarrenarbeitern, die sämtlich in höherem Alter eingetreten sind: rom Beginn bis zum Ende der 50 er Jahre. Sofern es sich, z. B. bei den Steinhauern, um echte Phthisen handelt, erscheint die Annahme einer Spätinfektion nicht ungerechtfertigt. Sodann ist eine Reihe von Todesfällen zu erwähnen, 23 an der Zahl, die fast sämtlich in den gleichen Fabrikbetrieben - Schuhwarenund Holzbearbeitungsfabriken - sich ereignet haben, bezüglich deren die Angehörigen ganz spontan äußerten: „Da gibts ja immer Gelegenheit sich anzustecken". Auch diese Fälle sind nach meiner Überzeugung zum 
großen Teil als exogen entstanden aufzufassen, da wir auch für sie keine auffindbare Infektionsgelegenheit in der Jugend haben. Nach den Altersklassen verteilen sie sich auf folgende Jahre:

\begin{tabular}{c|c|c|c|c|c}
\hline 21-25 Jahre & $26-30$ Jahre & $31-35$ Jahre & $36-40$ Jahre & $41-45$ Jahre & $56-65$ Jahre \\
\hline 2 & 2 & 13 & 3 & 2 & 1
\end{tabular}

Bemerkenswert erscheint die erhebliche Zunahme in der Altersstufe 31 bis 40 Jahre; gerade aus diesem Faktum kann nach meinem Dafürhalten auf eine stattgehabte exogene Infektion geschlossen werden, die man wohl nicht mit Unrecht in die ersten Jahre nach dem Verlassen des Elternhauses und der Beschäftigung in den betreffenden Betrieben verlegt. Wäre eine Kindheitsinfektion der Ausgangspunkt, die spätere Fabrikarbeit nur das schädigende auslösende Moment, so würde zu erwarten sein, daß die Todesfälle sïh weniger auf einen zeitlich eng begrenzten Altersabschnitt zusammendrängen, als vielmehr auf eine gröBere bald nach der Pubertāt einsetzende Periode verteilen würden. Außerdem müBten, da alle Arbeiter als infantil infiziert anzusehen sind, bei Einwirkung der gleichen Schädlichkeiten selbst unter Berücksichtigung aller individuellen Besonderheiten verhältnismäBig zahlreichere Arbeiter eines Betriebes erkranken, als es tatsächlich geschieht. Indes ist die Zahl dieser Gruppe von Todesfällen zu klein, als daB man aus der Verteilung auf die einzelnen Altersklassen begründete weitergehende Schlüsse bezüglich Art und Zeit der Reinfektion ziehen könnte.

Es blieben jene Fälle mit unbekannter Infektionsquelle kurz zu erwähnen übrig, 171 an Zahl, die entweder gewissermaßen als erste Fälle in einer Familie auftauchten und eine oder mehrere Erkrankungen nach sich zogen, oder die mehrere Familienmitglieder ohne nachweislichen gegenseitigen Zusammenhang betrafen. Sie verteilen sich auf folgende Altersklassen:

6-10 Jahre $16-20 \mathrm{~J} .21-25 \mathrm{~J} .|26-30 \mathrm{~J} .31-35 \mathrm{~J} .36-40 \mathrm{~J} .41-45 \mathrm{~J}| 46-.55 \mathrm{~J} .56-65$ J. 66-75 J. 1 12 18 27 26 13 14 33 22 5

Irgendwelche Besonderheiten bieten sie im übrigen für die Lösung der vorliegenden Frage nicht.

Ist also von den eben kurz besprochenen Fällen mit nicht nachweisbarer Infektionsquelle zum mindesten ein erheblicher Teil wahrscheinlich nichtendogenen Ursprungs, so lieB sich bei einer großen Reihe weiterer Fälle exogene Infektion als sehr wahrscheinlich oder sicher vorliegend 
ermitteln. Gerade diese Gruppe von Fällen, 189 an Zahl = 26.9 г. H. der Gesamtzahl, darf besondere Beachtung beanspruchen, da sie in der Hauptsache solche erwachsene Individuen umfaBt, die in mehr oder weniger unmittelbarem AnschluB an längeren Verkehr mit Tuberkulösen selber an Phthise erkrankten. Hierzu kommt eine kleine Zahl von Wohnungs- bzw. Werkstätteninfektionen. Das Alter der Verstorbenen verteilt sich auf folgende Jahre:

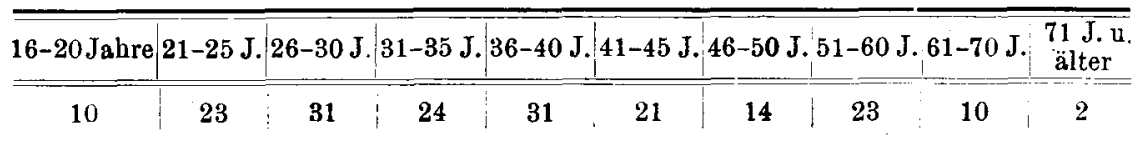

Man findet also neben der stärksten Beteiligung der Altersklassen 20 bis 40 Jahre auch noch in den höheren Stufen ganz ansehnliche Ziffern, z. B. in der Zeit von 51 bis 70 Jahren noch 33 Todesfälle = ca. $1 / 6$ der Gesamtzahl. Als Infektionsquellen kommen in Betracht (in Verhältnisziffern):

\begin{tabular}{|c|c|c|c|c|c|c|c|c|}
\hline Ehegatte & Eltern & $\begin{array}{c}\text { Ge- } \\
\text { schwister }\end{array}$ & Kinder & Freunde & Wohnung & $\begin{array}{l}\text { Werk- } \\
\text { statt }\end{array}$ & $\begin{array}{c}\text { Ver- } \\
\text { wandte }\end{array}$ & $\begin{array}{l}\text { Stellung } \\
\text { in tuberk. } \\
\text { Familien }\end{array}$ \\
\hline $19 \cdot 3$ & $14 \cdot 2$ & $17 \cdot 4$ & 20.5 & $3 \cdot 1$ & $25 \cdot 2$ & $7 \cdot 9$ & $6 \cdot 3$ & $4 \cdot 7$ \\
\hline
\end{tabular}

Beachtenswert ist die Bedeutung der Wohnung als Infektionsquelle, die für das hiesige Material die Hauptrolle spielt, von anderer Seite freilich (Römer) etwas skeptisch bewertet wird. Selbstredend kann man, besonders hinsichtlich dieses Faktors, stets nur in dem Sinne einer mehr oder weniger groBen Wahrscheinlichkeit reden, aber es gibt doch Fälle, in denen er derart nahe liegt, daß an ihm vorübergehen fast hieße, ihn nicht sehen wollen. Einige Beispiele nur sei mir in aller Kürze mitzuteilen gestattet. Ein hiesiger Oberwachtmeister, dessen nähere und weitere Familie durchaus frei von Tuberkulose ist, zieht 1909 - er war bis dahin auf dem Lande stationiert - infolge Versetzung nach Zeitz hier in die undesinfizierte Wohnung seines unmittelbaren Vorgängers, der etwa $1 \mathrm{Jahr}$ lang an schwerer Lungen-, Kehlkopf- und Darmtuberkulose krank gelegen und 4 Wochen nach seinem Wegzug starb. 2 Jahre später (1911) erkrankte die aus absolut gesunder Familie stammende, bis dahin kerngesunde Frau des Erstgenannten an Lungentuberkulose. Sollte hier nicht Wohnungsinfektion vorliegen? Ein anderer Fall: Eine völlig gesunde Fleischermeisterswitwe aus einem Nachbarort, die ihren Mann an Tuberkulose verloren, nimmt sich einen Gesellen und knüpft mit ihm ein Liebesverhältnis an. Er bezieht Raum und Bett des Verstorbenen. Nach 
wenigen Jahren erkrankt er an Schwindsucht, das Verhältnis wird gelöst, der Geselle entlassen. Die Frau findet bald Ersatz in einem neuen Gehilfen und heiratet diesen aus gesunder Familie stammenden Liebhaber. Nach einiger Zeit erkrankt auch er an Tuberkulose und stirbt 1902 im Alter ron 38 Jahren. Noch ein drittes ebenso bezeichnendes Beispiel sei mir anzuführen erlaubt: Es handelt sich um eine absolut gesunde, wohlhabende alteingesessene Gutsbesitzersfamilie meines Kreises. Ein Sohn kommt, 15 Jahre alt, in meinen jetzigen Wohnort zwecks Besuchs des Gymnasiums zu einer Familie in Pension, in der kurz vorher der Schwiegersohn, ein Lehrer aus einem nahen Dorfe, an Phthise bis zu seinem Tode gepflegt worden war. Er bezog das undesinfizierte Zimmer des Verstorbenen, benutzte das gleiche Bett, erkrankte bald darauf und starb nach 2 Jabren im Hause seiner Eltern. Nach seinem Tode schläft in dem gleichen Raum und Bett - der sehr verständige jetzt 83 jährige Vater betonte mir gegenüber dieses ausdrücklich unter Hinweis auf die damalige Unkenntnis von dem Wesen der Schwindsucht - ein jüngerer 14 Jahre alter Bruder, wird nicht lange darauf krank und stirbt 1884 . Wiederum wird das gleiche Zimmer und Bett sodann von einer älteren Tochter benutzt, die sich später verheiratete und nach 7 Jahren 189128 Jahre alt gleichfalls an Phthise zugrunde geht. Möge der zweite und dritte Todesfall auch auf direkten Kontakt zurūckzuführen sein, so beruhen der erste sowohl wie die beiden vorher skizzierten Fälle nach meiner Uberzeugung unzweifelhaft auf Wohnungsinfektion. Weitere typische Fälle anzuführen, würde nicht interessieren; jedenfalls dürfte es berechtigt sein, bei derartigem Zutageliegen von Ursache und Wirkung die exogene Entstehung der betreffenden Phthisen für das Nächstliegende und Wahrscheinlichste zu halten. - DaB bei Erwachsenen die Kinder häufiger zur Infektionsquelle für die Eltern werden als umgekehrt, ist recht bemerkenswert und ein Hinweis für die exogene Entstehung solcher Fälle. Ein charakteristisches Beispiel nur sei mitgeteilt: Das $15^{1} / \mathbf{3}$ jährige Mädchen H. starb im Jahre 1901, eine 28 jährige Schwester, die sich an der eben genannten zweifellos infiziert hatte, 1904 an Phthise. Die Mutter - der Vater sowohl wie die gesamte weitere Familie leben und sind gesund - hatte beide Kinder gepflegt, erkrankte einige Jahre später und starb 1912 im Alter von 65 Jahren ebenfalls an Tuberkulose. Es würde zu weit führen, wollte ich noch sonstige gleichlautende Beispiele mitteilen; aus allen geht mit einer fast an Sicherheit grenzenden Wahrscheinlichkeit hervor, daB die Krankheit durch exogene Infektion entstanden ist. Nun ist gegen diesen Infektionsmodus eingewendet worden, er sei schon deshalb sehr unwahrscheinlich, weil bei Erkrankung und Tod eines Fhegatten an Tuberkulose selten oder nie Infektion des anderen erfolge; z. B. behauptet ein 
so erfahrener Tuberkulosearzt wie Prof. Petruschky, auf den sich auch Römer bezieht, er habe eine solche Infektion nie beobachtet. Auch ich muB gestehen, wenn ich meine frühere sehr umfangreiche praktische Tätigkeit überschaue, mich nicht zu erinnern, je einen derartigen Fall erlebt zu haben. Wahrscheinlich beruht dies darauf, dab der praktische Arzt wohl nur selten in die Lage kommt, derartige Doppelinfektionen, die vielleicht eine längere Reihe von Jahren auseinander liegen und von verschiedenen Ärzten behandelt wurden, zu beobachten. Jedenfalls habe ich in dem erhaltenen Material unter 265 Fällen ron Erkrankung und Tod eines Ehegatten an Tuberkulose eine Anzahl, ich kann wohl sagen ziemlich sicherer Fälle von Ansteckung des anderen Ehegatten am zuerst verstorbenen feststellen können: 216 Ehegatten waren zur Zeit der Ermittelungen noch gesund, 11 weitere an sonstigen Krankheiten und 38 gleichfalls an Lungenschwindsucht gestorben, was einem Verhältnis von 84.7:14.3 entspricht. Daß ein Teil der zurzeit Gesunden bei der Kürze der nach dem Tode des anderen Ehegatten verflossenen Frist nicht noch später an Phthise erkrankt, ist wohl nicht zweifelhaft, dagegen läßt sich von einer erheblichen Zahl annehmen, daß das Zusammenleben mit dem Kranken ihnen keine Infektion eingetragen hat. Die nachstehenden Übersichten geben eine ziffernmäBige Darstellung der Verhältnisse:

a) Nach dem Tode eines Ehegatten blieb der andere gesund:

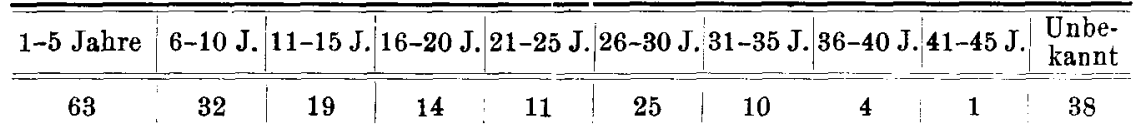

b) Die Zwischenzeit zwischen dem an Tuberkulose erfolgten Tode beider Ehegatten betrug:

\begin{tabular}{|c|c|c|c|c|c|}
\hline $1-5$ Jahre & $6-10$ Jahre & 11-15 Jahre & 16-20 Jahre & $21-25$ Jahre & Unbekannt \\
\hline 20 & 10 & 1 & 2 & 1 & 4 \\
\hline
\end{tabular}

Danach kann man wohl sagen, daB, wenn 10 Jahre nach dem Tode eines Ehegatten verflossen und der andere noch nicht an Tuberkulose erkrankt ist, dieser auch sehr wahrscheinlich von einer Infektion durch jenen frei geblieben ist. - Einige bezeichnende Beispiele von Gatteninfektion seien mitgeteilt: Die 28 jährige Frau eines Eisenbahnbeamten, deren Eltern, sieben Geschwister und Verwandte gesund sind, stirbt im Jahre 1894 in Trebnitz, Kreis Weißenfels, an Phthise. Nach ihrem Tode bittet der Mann, in dessen Familie gleichfalls alles gesund, der bei wieder- 
holten bahnärztlichen Untersuchungen frei von Tuberkuloseverdacht befunden worden ist, um Versetzung und kommt nach Zeitz. Hier erkrankt und stirbt er 1903 im Alter von 45 Jahren gleichfalls an Schwindsucht. Es wäre schwer, nicht anzunehmen, daB er sich an seiner Frau infiziert habe. Ein anderer Fall: Der Weber R. stirbt 1894 in Zeitz im Alter ron 57 Jahren an Tuberkulose, nachdem ihn seine Frau während 3jährigen Siechtums gepflegt. Nach seinem Tode verläBt sie die Wohnung und bezieht in einem entfernten Stadtviertel eine andere; hier erbrankt und stirbt sie an der gleichen Krankheit im Jahre 1912 im 75. Lebensjahre. An eine Autoreinfektion auf der Basis einer erlittenen Jugendinfektion kann bei der 75jährigen kaum geglaubt werden. SchlieBlich sei noch folgendes Beispiel mitgeteilt: Ein 49jähriger Ofenwärter, aus vollkommen gesunder Familie stammend, stirbt 1907 an Schwindsucht; seine Frau, dje ihn bis zum Tode gepflegt, wechselt danach die Wohnung, erkrankt bald darauf in ihrem neuen Logis und stirbt, 55 Jahre alt, 1912 auch an Phthise. Die Annahme einer Gatteninfektion lä日t sich wohl kaum von der Hand weisen. Weitere Mitteilung von Belegen für Gatteninfeltion würde den Rahmen dieser Erörterungen überschreiten; soviel steht jedenfalls fest, daß sie vorkommen und nicht $z u$ selten sind. Warum in der überwiegenden Mehrzahl der andere Gatte nicht infiziert wird, ist einwandfrei noch nicht erklärt. Die Annahme von Immunitätsvorgängen im Sinne Römers liegt ja nahe, die aber niemals eine restlose Aufklärung gewähren; vor allem bleibt der Punkt unverständlich, daß der in der Jugend erhaltene Schutz, welcher doch nur ein relativer und nur leichteren Infektionen gegenüber wirksam sein soll, so häufig sich gerade bei schweren Infektionsgelegenheiten, wie sie Gattenerkrankungen sind, aktiv zeigt, während er in vielen anderen Fällen, bei denen es sich um leichtere und mehr vorübergehende Infektionschancen handelt, versagt. Ich erkläre mir die geschilderten Verhältnisse so, daß es sich bei dem längeren Zusammenleben mit einem phthisischen Gatten um eine fortgesetzte, allmählich sich steigernde Immunisierung des anderen Ehegatten mit lebenden Tuberkelbazillen handelt, indem im Anfang der Krankheit, also zu einer Zeit, in der relativ wenig Bazillen ausgeschieden werden, nur solche Mengen aufgenommen werden, die unterhalb der krankmachenden Dosis liegen; nach und nach findet vermehrte Aufnahme von Bazillen statt, die aber vom Organismus überwunden werden. Bleibt in selteneren Fällen die allmähliche Immunisierung aus und werden von vornherein krankmachende Mengen von Tuberkulosevirus aufgenommen, so erkrankt der andere Ehegatte. Ob eine solche Erklärung zutreffend ist, sei dahingestellt. - Jedenfalls bleibt trotz aller Fortschritte, die wir gerade bezüglich der Kenntnis der Tuberkulose gemacht haben, noch vieles zu klären übrig. 
Nunmehr will ich kurz zu denjenigen Todesfällen übergehen, welche sehr wahrscheinlich als auf infantiler Infektion beruhend anzusehen sind. Sie setzen sich fast ausschlieBlich aus Personen zusammen, die in der Kindheit mit schwindsüchtigen Angehörigen in Wohnungsgemeinschaft gelebt haben, also Gelegenheit zu sogenannter schwerer Infektion vollauf besaßen. Einzelne Fälle kommen hinzu, in denen, ohne daB familiäre Tuberkulose nachgewiesen, die Angehörigen mit Sicherheit angaben, daB nach Überstehen einer schwereren Noxe (meist einer ansteckenden Krankkeit) sich an dieselbe eine Kette von Krankheitserscheinungen anschlob, welche, mit kürzeren oder längeren Unterbrechungen auftretend, allmählich in Schwindsucht übergingen. Solche Fälle, in denen es sich lediglich um Mitteilungen über Ausschlag, Drüsen, Ohreiterungen und ähnliche Symptome handelte, sehe ich als in ihrer Genese unsicher an, da ja derartige Erscheinungen nicht immer der Ausdruck einer tuberkulösen Infektion zu sein brauchen; sie sind hier nicht in Rechnung gezogen. Ebenso bleiben sieben Fälle auBer Betracht, in denen außer der Frühinfektion noch Gelegenheit zur Spätinfektion gegeben war. Als sichere Frühinfektionen bleiben vor der Gesamtziffer 702 genau 101 Fälle $=14.3$ r. H. übrig. Es ist also nur ein rerbältnismäBig kleiner Teil aller Tuberkulösen, von denen man mit ziemlicher Wahrscheinlichkeit sagen kann, sie seien durch endogene Reinfektion entstanden. Positiv beweisen wird sich eine solche Annahme nie lassen, zumal im Hinblick darauf, daB die Zahl der gesund gebliebenen Geschwister, die der absolut gleichen Infektionsgelegenheit ausgesetzt gewesen, eine sehr viel größere ist; daher kehrt auf den Fragebogen die Ansicht immer wieder, die Schwindsucht könne nicht ansteckend sein. - Nicht uninteressant ist ein Überblick über das Alter, in welchem diese ,infantil schwer infizierten" Indiriduen gestorben sind:

\begin{tabular}{c|c|cc|c|c|c|c|c}
\hline \hline $1-5$ Jahre & $6-10 \mathrm{~J}$. & $11-15$ & $\mathrm{~J}$. & $16-20 \mathrm{~J}$. & $21-25 \mathrm{~J}$. & $26-30 \mathrm{~J}$. & $31-35 \mathrm{~J}$. & Zusammen \\
\hline \hline 11 & 12 & 9 & 25 & 29 & 11 & 4 & 101
\end{tabular}

Hieraus geht hervor, daB von keinem der in der Jugend nachweislich oder sehr wahrscheinlich schwer Infizierten die Mitte des 4. Lebensjahrzehnts überschritten wurde. Die große Mehrzahl starb am Ende des zweiten bzw. Anfang des 3. Jahrzehnts. Diese meines Erachtens wichtige Tatsache stimmt im wesentlichen mit den Ergebnissen überein, wie sie Dörner bei seinen Familienuntersuchungen in Liedolsheim für die gleiche Kategorie der Infizierten gefunden hat; er sagt: „Der EinfluB der elterlichen Tuberkulose auf die erwachsenen Nachkommen läßt erheblich nach und scheint nach dem 25. Lebensjahr, wenn sie nicht mehr in der Umgebung der Eltern leben, gar nicht mehr in 
Betracht zu kommen". Somit glaube ich, daB wir annehmen können, daß die später zur Phthise führende schwere Jugendinfektion im 2. oder 3. Lebensjahrzehnt manifest wird, d. h. also, daß Erkrankungen, die jenseits des 4 . oder 5. Jahrzehnts ausbrechen, mit gröBter Wahrscheinlichkeit mit einer infantilen Infektion keine ursächlichen Beziehungen mehr haben.

Sollte sich dieser Satz als richtig erweisen, dann kann man wohl zum mindesten für alle diejenigen Todesfälle mit unbekannter Infektionsquelle, die jenseits des 45. Lebensjahrs eingetreten sind, annehmen, daB sie nicht auf endogenen Reinfeltionen, sondern auf exogener Spätansteckung beruhen.

Nach diesen Ausführungen darf wohl als feststehend angesehen werden, daB der Satz, die Phthise des Erwachsenen sei das Produkt einer Autoreinfektion von einem infantilen Herd aus, in dieser Allgemeinheit unter keinen Umständen aufrecht zu erhalten ist. Sie ist mit groBer Wahrscheinlichkeit bald endogenen, bald exogenen Ursprungs; welche Quelle überwiegt, wird zum Teil von sozialen Verhältnissen, zur Hauptsache jedoch von den Maßnahmen abhängen, die wir im Kampf gegen die Tuberkulose treffen: Schutz der Kinder vor schwerer Infektion ist hier ebenso wichtig, wie Schutz der Erwachsenen vor den ihnen oft unbewuBten Infektionsgelegenheiten in und auBerhalb der Wohnung, des Hauses, der Betriebs- und Arbeitsstätte. Wünschenswert wäre es, wenn einige weitere Untersuchungen, wie die mitgeteilten, vorgenommen würden, um auf epidemiologischem Wege der Lösung noch mancher Frage, die klärungsbedürftig ist, nāher zu kommen. Das Tierexperiment, so hoch dessen Wert auch zu schätzen ist, und so sehr es uns eine tiefere Kenntnis der Tuberkulose, dieser Krankheit, die uns täglich neue Rätsel aufgibt, vermittelt hat, wird uns niemals allein über alle Fragen AufschluB zu bringen vermögen. Die biologische Methode bedarf notwendigerweise der Ergänzung durch die epidemiologische, die meines Erachtens in den letzten Jahren nicht ganz zu ihrem Rechte und voller Würdigung bei allen Autoren gekommen ist. Durch eingehendes Studium der Epidemiologie, d. h. der Experimente, die unsere größte Lehrmeisterin, die Natur, anstellt und zwar an ihrem vornehmsten Objekt, dem Menschen selber, werden wir nach meiner Überzeugung vor manchem Fehlweg bewahrt werden, in den uns Spekulation und Experiment zu leicht hineinführen; ihre Ergebnisse sollten stets das kritische Gegengewicht zu denen aller experimentellen Forschungen bilden. 